\title{
Striatopallidonigral Degeneration
}

\section{Report of a Case in a 15-year-old Girl}

\author{
W. E. BELL and W. F. McCORMICK \\ From the Departments of Paediatrics and Neurology, and Pathology (Neuropathology), University of Iowa College of \\ Medicine, Iowa City, Iowa 52240, U.S.A.
}

\begin{abstract}
Bell, W. E., and McCormick, W. F. (1971). Archives of Disease in Childhood, 46, 533. Striatopallidonigral degeneration. Report of a case in a 15-yearold girl. A 15-year-old girl is described with a sporadic, progressive illness manifested by unilateral limb rigidity and dystonia. Obvious dysarthria and some intellectual decline also were noted. Neuropathological findings included gross discoloration and shrinkage of the pallida and, microscopically, profound neuronal loss and gliosis of the caudata and putamena, with less severe neuronal loss from the pallida and substantia nigra. The disease bears some similarities to striatonigral degeneration, but certain clinical and morphological differences justify its consideration as a separate syndrome.
\end{abstract}

Degenerative disorders of the basal ganglia in childhood are unusual, and certain types remain as medical curiosities and aetiological enigmas. Huntington's chorea, Wilson's disease, and dystonia musculorum deformans are the most notable of the deep nuclear degenerative conditions occurring in children, and can generally be diagnosed before death with the appropriate clinical, genetic, and laboratory investigations.

The abnormalities identified in the basal ganglia of the patient described below appeared to us to be unique, as we could find no similar cases recorded in the childhood age group. In view of the anatomical location of the neuronal degenerative changes, we have chosen to refer to this disorder as striatopallidonigral degeneration. In this instance, the illness occurred in sporadic fashion and no aetiological clues became evident from the diagnostic assessment. The pathology in this case bears considerable resemblance to the adult cases desscribed by Adams, van Bogaert, and vander Eecken $(1961,1964)$ and referred to as striatonigral degeneration.

\section{Case Report}

This 15-year-old girl was admitted to University Hospitals for evaluation of spasmodic deviation of the left foot of 2 months' duration. Approximately one year before the onset of symptoms she had been noted to become more sullen and withdrawn, with periodic crying

Received 14 December 1970. spells. With the beginning of the school year 6 months before admission, her academic achievement was noted to be poorer than in previous years and her personality problems continued. She retained few friendships and preferred to remain alone rather than to engage in social relationships with her school colleagues.

Two months before admission, she developed recurrent episodes of medial deviation of the left foot, which interfered with walking and eventually required the use of crutches. After a few days, the distorted posture of the left foot became more persistent and was aggravated by weight bearing or tactile stimulation of the extremity. Three weeks before admission, similar episodic, dystonic postural changes appeared in the left upper extremity, and these likewise became progressively worse in severity. The development of intense pain associated with the rigidity and recurrent spasms led to hospital admission. The child's mother had observed that during sleep no postural or dystonic abnormalities were present, and that during waking hours either excitement or tactile stimulation of the left arm or leg would evoke dystonic spasms. During the months before entry to the hospital, weight loss of $15.4 \mathrm{~kg}$ had occurred. There was no family history of similar neurological disorders.

On examination, she was a slender girl who looked chronically ill and obviously uncomfortable. Blood pressure was $92 / 62 \mathrm{mmHg}$. The neck was supple. Auscultation of the heart and palpation of the abdomen revealed no abnormalities. The pupils were equal and reacted to light. Extraocular movements were intact and visual fields normal to confrontation. KayserFleischer rings were not present. Sensory examination was normal. Her speech was moderately dysarthric, 
and movement of the tongue was diminished. Periodically she would burst into tears and complain of pain in the left arm and leg. Except when crying, her face was expressionless, with drooling of saliva. Profound rigidity was present in the left arm and leg, which fixed the limbs in extension at the elbow and the knee and which was increased with any attempt by the examiner to flex the limbs at these joints. Dystonic deformities on the left resulted in bizarre postures with the elbow rigidly extended, the forearm markedly pronated with intense flexion at the wrist (Fig. 1 and 2), and with the

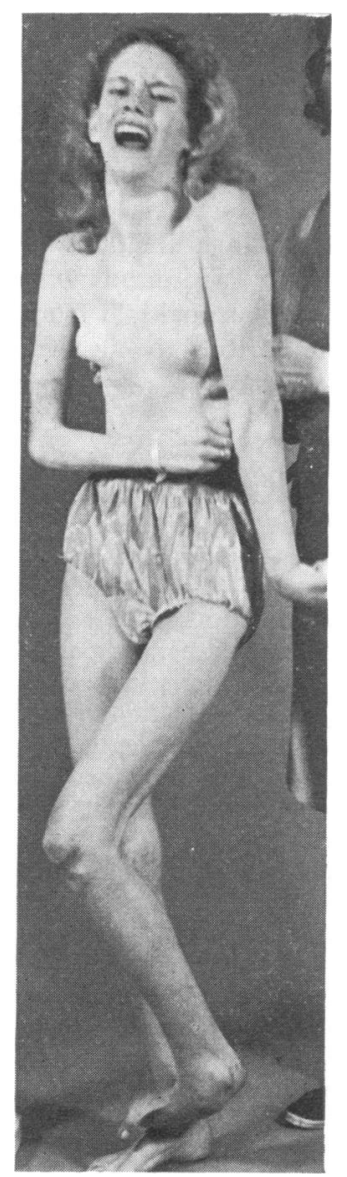

FIG. 1.-Rigidity of left arm and leg with painful dystonia producing equinovarus deformity of the foot and pronation of the forearm with flexion of the wrist and fingers. Painful aspect of spasm reflected by patient's facial expression.

foot held in an equinovarus position with fixed extension of the great toe. The left lower extremity was either held in rigid extension or, with painful spasmodic episodes, partially flexed at the knee. Muscle power could not be adequately assessed on the left side though it did not appear to be reduced. Deep tendon reflexes in the left lower extremity were moderately increased. No tremor or ataxia was noted, but holding the arms outstretched aggravated the dystonic posturing. Muscle tone, power, and reflexes of the right arm and leg were entirely normal. Her gait was slow, with dragging of the rigid left leg and with the distorted left hand carried in a protective fashion in the palm of the right. Significant muscle atrophy or fasciculations were not observed.

Laboratory studies included a normal urinalysis and

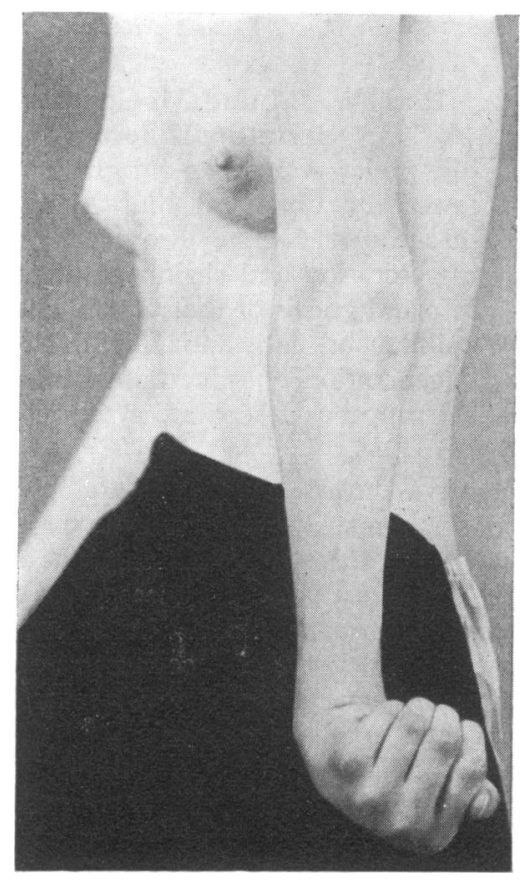

FIG. 2.-Postural disturbance of the left upper limb during dystonic episode.

blood count. Sedimentation rate was $16 \mathrm{~mm}$ per hour. BUN, serum electrolytes, calcium, and blood sugar were normal. Serum caeruloplasmin was 391 units ( $n=280-570$ units). Urine copper was $13 \mu \mathrm{g} / 24$ hours. $X$-rays of the skull and chest were normal and a pneumoencephalogram showed good filling of the ventricular system with no abnormalities. CSF protein was $54 \mathrm{mg} / 100 \mathrm{ml}$, glucose $65 \mathrm{mg} / 100 \mathrm{ml}$. Motor nerve conduction velocities in upper and lower limbs were normal. A radioactive brain scan showed no areas of abnormal uptake. An EEG shortly after admission was abnormal, with occasional bursts of 6 per second spikes and some diffuse theta slowing. When repeated one month later, the record showed progressive worsening with more high voltage slow activity bilaterally. A psychometric examination revealed a full-scale IQ of 83. 
During the first 3 weeks in hospital, the rigidity and dystonia of the left arm and leg became progressively worse as did the pain in the corresponding limbs. During this period, diphenylhydantoin, diazepam, chlorpromazine, and haloperidol had been tried alternately, but none diminished the rigidity of dystonic spasms. She then complained of intermittent diplopia and a few days later had 2 grand mal seizures.

During the sixth and last week of hospital stay, the postural disturbance of the left arm and leg became persistent throughout the waking hours but still was worsened by movement or tactile stimulation. She stopped walking and preferred to remain in bed unless forced to stand. She returned to the outpatient clinic 6 weeks after discharge. Her neurological findings were essentially unchanged except for bilateral extensor plantar responses and increased quadriceps stretch reflexes bilaterally. Pain in the left arm and leg was less than before. Her illness slowly progressed and death occurred at home 5 months after admission to the hospital.

Necropsy. This was limited to the brain and spinal cord. The leptomeninges were normal in appearance and the gross observation of the uncut brain was not remarkable. Serial section of the cerebral hemispheres revealed normal demarcation between grey and white matter with well-preserved convolutional and central white matter. The ventricular system was of normal size and was lined by smooth, glistening ependyma. The globi pallidi were shrunken and revealed a pronounced yellow discoloration (Fig. 3). The claustra, caudata, and putamena were grossly normal, as were the thalami and subthalamic nuclei.

Microscopical examination of the cerebral cortex revealed normal numbers and structure of neurones. The intraparenchymal vessels generally were normal

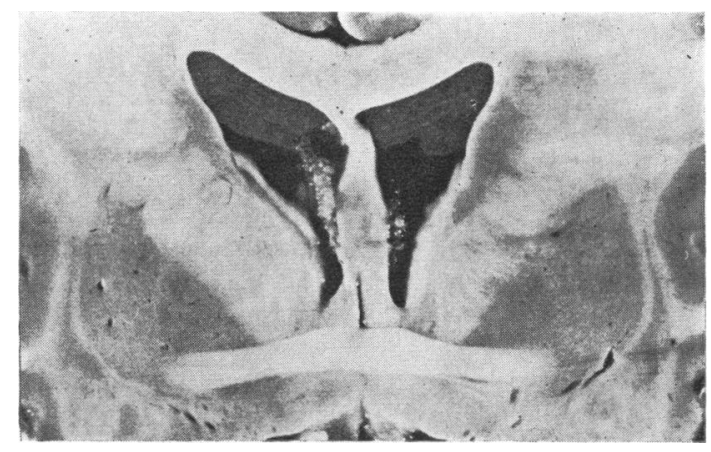

FIG. 3.-Gross photograph at the level of the anterior commissure illustrating dark pigmentation of the globi pallidi without cavitation. The caudata and putamena appear grossly normal.

but a few small vessels, chiefly veins, in the striata were cuffed by mononuclear cells. No abnormal accumulation of iron or calcium was observed in the basal ganglia. Severe neuronal loss was present in the caudata and putamena bilaterally (Fig. 4 and 5) and moderate depletion of neurones was noted in the globi pallidi. The few remaining nerve cells in the pallidal region contained moderate quantities of PAS-positive lipofuscin. Neuroaxonal dystrophy was not present. There was intense astrogliosis in the caudate nuclei, putamena, and moderate gliosis in the globi pallidi. Neither neuronal inclusions nor a storage material were found. Hypermyelinization of the involved nuclear groups was not present, and myelinization of internal capsule, anterior commissure, and optic tracts appeared normal. Neuronal loss was also present in the reticular zone of the substantia nigra with a moderate degree of

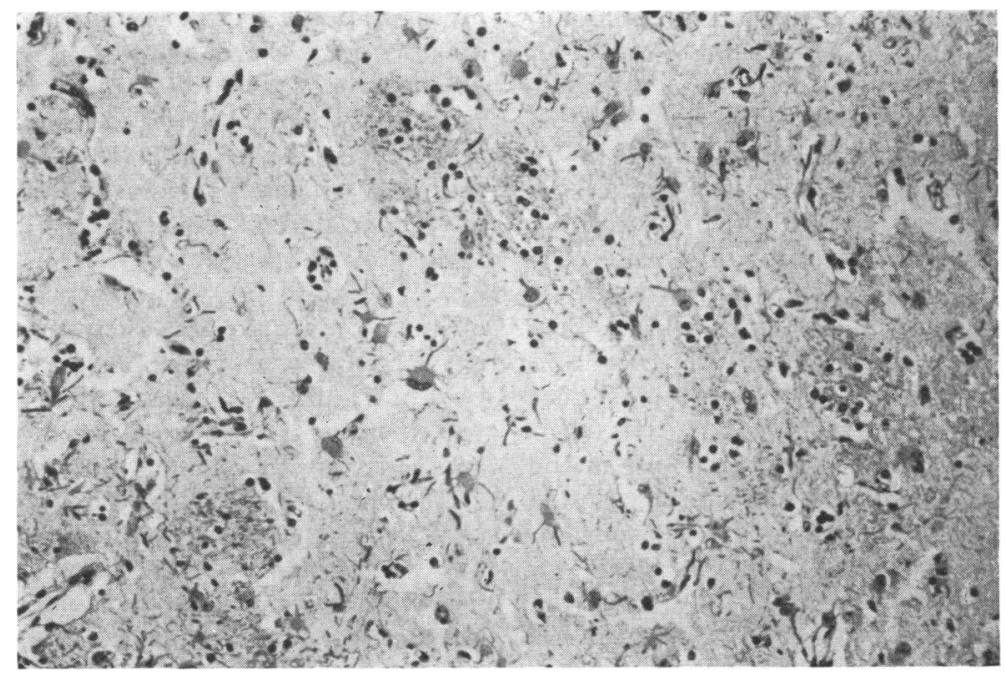

FIG. 4.-Photomicrograph of globus pallidus illustrating neuronal loss and extensive gliosis. (PTAH $\times 150$. 


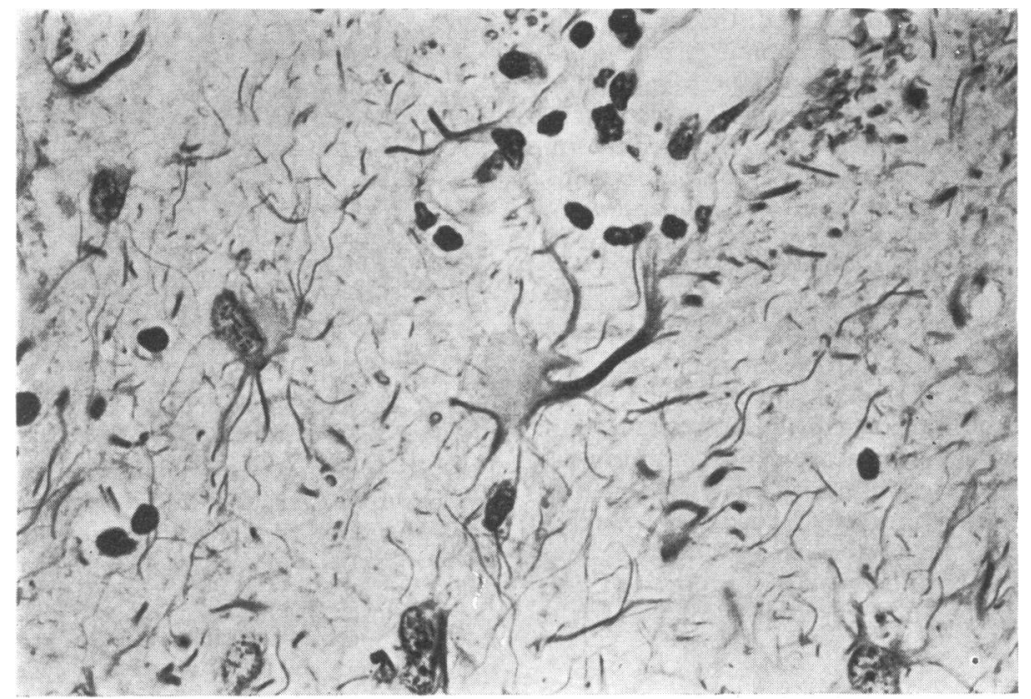

Fig. 5.-Photomicrograph of caudate nucleus illustrating intense reactive gliosis. (PTAH. $\times 500$.)

replacement gliosis. The subthalamic nuclei were normal, and myelinization within the brainstem was well preserved. In the cerebellum, there was mild focal loss of Purkinje cells with hyperplasia of Bergmann glia. The spinal cord revealed no abnormalities.

\section{Discussion}

The initial clinical manifestations of this illness were sufficiently bizarre for hysteria to be considered. The profound rigidity did not change under hypnosis and the subsequent development of diplopia and seizures rendered conversion reaction implausible. Local tetanus was entertained in differential diagnosis due to the painful aspect of the illness and because of the precipitation of muscle spasm by tactile stimulation. Both arm and leg on the same side would not be expected to be involved in this variant of tetanus. The pathology, also, is clearly incompatible with that possibility. For the same reason, a postencephalitic state with dystonia seems excluded. Intoxications such as carbon monoxide, manganese, and cyanide may produce basal ganglia disease, but the gross and microscopical pathology of these conditions does not resemble that observed in this case. In addition, her illness continued to progress while in the hospital for 6 weeks during which continued exposure to toxic material would not have occurred.

The absence of involvement of the neocortex plus the isolated occurrence of the illness in only one member of the family provided additional evidence excluding Huntington's chorea. Likewise, the normal serum caeruloplasmin and urine copper, the absence of cavitary lesions in the striatum and the lack of Alzheimer glial cell changes made Wilson's disease unlikely.

When the brain was sectioned and observed grossly, the shrunken, discoloured appearance of the globi pallidi suggested Hallervorden-Spatz disease. However, the pathological hallmarks of this disease, including iron deposition in the pallidum and neuroaxonal dystrophy, were not present in our case. While extrapyramidal manifestations are prominent in patients with Hallervorden-Spatz disease, the rapidity of progression of the disease noted in the case described above would be most unusual. The rapid clinical course would also be most atypical in dystonia musculorum deformans. Truncal involvement is usually present, intellect remains unaffected, and the EEG is usually normal in this disease, all being features different from our patient. Zeman and Dyken (1968) and Zeman (1970) denied the existence of an established neuropathological pattern in dystonia musculorum deformans and did not describe findings resembling those in the striatum, pallidum, and substantia nigra in our case.

Remaining possibilities in differential diagnosis are a group of poorly understood disorders of unknown aetiology. These include progressive pallidal degeneration, pallidoluysian atrophy, familial pallidonigral degeneration, and striatonigral degeneration. It it with this category of primary degenerations that our case seems to bear the closest similarity. Progressive pallidal degeneration described by Hunt (1917) was characterized by more 
profound changes in the pallidum than in other basal nuclei. The substantia nigra was not involved in these cases which clinically were classified as juvenile 'paralysis agitans'. van Bogaert (1946) subsequently identified cases with similar pallidal changes, but also with degeneration of the subthalamic nuclei, a condition referred to as 'pallidoluysian atrophy'. Clinical features of this disorder included dystonia and progressive rigidity accompanied by torticollis, tremor, and, on occasion, ballistic movements. Progressive pallidal degeneration of Hunt was associated with equinovarus deformities of the feet with limb rigidity and tremor followed by nonpatterned truncal dystonic movements. Speech and swallowing disturbances occurred early in the course of this illness though intellect remained unimpaired. Degeneration of the pallidonigral system reported by McCormick and Lemmi (1965) appeared considerably different from our case both in its clinical and morphological features. Cavitary degeneration of the pallidum and substantia nigra with severe damage of the corpus Luysii in these cases stands in sharp contrast to the findings in our case.

From a morphological standpoint, the abnormalities in our case share more common features with striatonigral degeneration than with any of the previously mentioned entities. Adams et al. (1961, 1964) described a group of patients with clinical findings consistent with Parkinson's disease, but in whom pathological abnormalities included neuronal degeneration of the striatum and substantia nigra. The 4 patients were adults with onset of symptoms in the 5 th to the 7 th decades. Death occurred from 3 to 7 years after onset, usually from intercurrent disease. The initial clinical symptoms were usually rigidity and slowness of movement. With progression of the disease, speech and swallowing disturbances occurred, and 2 of the 4 cases developed tremor. None of these patients exhibited dystonia, chorea, or athetosis. The authors chose the term striatonigral degeneration because of the distribution of the pathological process. Atrophy and brownish discoloration of the putamena were observed, in addition to visible depigmentation of the substantia nigra. The most severe neuronal loss was noted in the putamen, caudate, and substantia nigra, with marked gliosis in the involved areas. Neurones of the globus pallidus were almost totally spared, though grossly this structure appeared diminished in volume. This volumetric alteration of the globus pallidus was attributed to loss of striatopallidal fibres. One case showed some neuronal loss in the corpus Luysii and one revealed Lewy inclusion bodies in certain cranial nerve nuclei.
An additional adult case was described by Mayo and Barron (1966) and 4 more have recently been reported by Andrews, Terry, and Spataro (1970). The latter 4 patients also were adults in their 6th or 7th decade of life, and, as with all cases previously described, the illness occurred in sporadic fashion. 3 of these patients underwent stereotaxic thalamectomy with a beneficial response resulting in each. As in the previously identified cases of striatonigral degeneration, brown discoloration of the putamen and pallor of the substantia nigra with profound neuronal loss from these regions was found pathologically.

Several differences are apparent, both clinically and pathologically, in our case compared to those recorded in the literature under the designation of striatonigral degeneration. All previous cases have occurred in middle-aged or elderly adults, while our patient was only 15 years of age. Rigidity and painful dystonia were the outstanding features of her illness and tremor was never present. The rapid progression culminating in death within months after the onset of motor deficits is unlike previously reported cases. Visible atrophy and discoloration of the putamen, so notable in described cases of striatonigral degeneration, were not found in our patient. The putamena, in fact, appeared grossly normal, though microscopically were extensively damaged. The pallida did show obvious discoloration, unlike those described by Adams et al. (1964) and Andrews et al. (1970). The caudate in the present case contained severe neuronal loss and gliosis. The degree of this alteration appears greater in severity than in the reported cases of striatonigral degeneration. In addition, the description of lipofuscin pigmentation of variable degree within the involved putamena was not observed in our case.

In view of the clinical and morphological characteristics of this case, we feel that it deserves the designation of striatopallidonigral degeneration. We have not found a previously recorded case in childhood like this. While it shares certain pathological characteristics with striatonigral degeneration, the differences seem sufficient to consider it as a separate syndrome. The paradox of the predominantly unilateral limb involvement, despite the bilateral and nearly symmetrical structural abnormalities of the striatum, pallidum, and substantia nigra, remains unexplained.

\section{REFERENCES}

Adams, R. D., van Bogaert, L., and vander Eecken, H. (1961). Dégenérescences nigro-striées et cerebello-nigro-striées. Psychiatria et Neurologia, 142, 219. 
Adams, R. D., van Bogaert, L., and vander Eecken, H. (1964). Striatonigral degeneration. Fournal of Neuropathology and Experimental Neurology, 23, 584.

Andrews, J. M., Terry, R. D., and Spataro, J. (1970). Striatonigral degeneration. Archives of Neurology, 23, 319.

van Bogaert, L. (1946). Aspects cliniques et pathologiques des atrophies pallidales et pallido-luysiennes progressives. fournal of Neurology, Neurosurgery and Psychiatry, 8, 125.

Hunt, J. R. (1917). Progressive atrophy of the globus pallidus. Brain, 40, 58.

McCormick, W. F., and Lemmi, H. (1965). Familial degeneration of the pallidonigral system. Neurology, 15, 141.

Mayo, G. M., and Barron, K. D. (1966). Striato-nigral degenera- tion. fournal of Neuropathology and Experimental Neurology, 25, 172.

Zeman, W. (1970). Pathology of the torsion dystonias (dystonia musculorum deformans). Neurology, 20, 79.

Zeman, W., and Dyken, P. (1968). Dystonia musculorum deformans. In Handbook of Clinical Neurology, vol. 6, p. 517. Ed. by P. J. Vinken and G. M. Bruyn. North Holland Publishing Co., Amsterdam.

Correspondence to Dr. W. E. Bell, Departments of Pediatrics and Neurology, University Hospitals, Iowa City, Iowa 52240, U.S.A. 